\title{
«LA COGNIZIONE DEL DOLORE» DI CARLO EMILIO GADDA: IL «ROMANZO FAMILIARE» DI UNA VITA
}

\author{
Nota del s.c. CLELIA MARTIGNONI (*)
}

(Adunanza del 3 ottobre 2019)

SuNTO. - Il saggio intende indagare La cognizione del dolore di Carlo Emilio Gadda come "romanzo familiare" protratto per quasi una vita. Una chiave di indagine per l'universo gaddiano e il romanzo suddetto è la «complessità» acquisita da Gadda negli studi filosofici universitari, e presente in tutti gli aspetti della sua poetica e produzione, delle sue narrazioni (sempre incompiute), sia sul piano delle ossessioni tematiche sia nel trattamento e nelle tecniche narrative e formali, sempre plurali, ibride, combinatorie, molteplici.

ABSTRACT. - This essay aims at analysing La cognizione del dolore as a "family novel" which was continued by Carlo Emilio Gadda for nearly all his life. A key element of this investigation is the notion of "complexity" which Gadda acquired during his university studies in the field of philosophy and which is present in his poetics and many aspects of his literary output. Such a notion exerts a strong influence on the obsessively recurring themes as well as the manifold formal and narrative techniques which are constantly hybrid and based on combinatorial mechanisms.

\section{L'EDIZIONE TORMENTATA DELLA «COGNIZIONE»} (1938-1963 E OLTRE)

Ogni volta che si riprende in mano La cognizione del dolore di Carlo Emilio Gadda (1893-1973), uno degli scrittori più potenti - e più

(*) Università degli Studi di Pavia, Italy. E-mail: clelia.martignoni@unipv.it 
milanesi - del Novecento, se ne risente il tumultuoso fascino, cui contribuiscono molti elementi: la forza della visione e del linguaggio, e la compresenza tumultuosa di più registri: tragico, malinconico, satirico, lirico, e di più stili: aulico-solenne e popolare-dialettale.

Il racconto è imperniato sulle tensioni affettive di una famiglia, dal cognome grottesco Pirobutirro - che, commisurato ai drammi narrati, segnala di per sé la contaminazione di stili - nel paese brianzolo di Longone, o Lukones, travestito da fittizio Sudamerica, e nella decaduta dimora familiare, luogo di oscuri conflitti e sofferenze. Siamo nell'estate del 1934 (o, come si legge bizzarramente in un punto, del '33):' in epoca fascista, cui alludono alcuni riferimenti, come l'infido e vessatorio "Ninstitúo de vigilancia para la noche". Protagonisti: una madre anziana, la mesta «signora», provata dalla guerra recente (la prima guerra mondiale) che le ha inflitto la perdita del figlio minore amatissimo, evocato di continuo; e il figlio superstite, Gonzalo (nome di ascendenza shakespeariana), alter ego dell'autore: ingegnere, ma appassionato soprattutto di lettere e filosofia, strambo, introverso, nevrotico, aggressivo, mal visto dai popolani di Lukones. Altri personaggi li attorniano: il medico sempliciotto che ha in cura madre e figlio; il gruppetto invadente di servitori e domestici, inviso a Gonzalo; la guardia del Ninstitúo-servizio di sicurezza, il truffaldino Gaetano Palumbo/Manganones, finto sordo di guerra (come si svela via via dalle ricostruzioni del Colonnello Di Pascuale). Culmine drammatico della vicenda è l'orribile assassinio notturno della «signora», insoluto.

Limito al minimo in questa sede i pur necessari chiarimenti preliminari sulle vicende compositive dell'opera, sempre incompiuta: esce in sette puntate (che l'estroso Gadda chiama "tratti") tra 1938-'41 sulla rivista fiorentina «Letteratura»; ed è raccolta in volume ancora inconclusa ma con rielaborazione variantistica nel 1963 per Einaudi. ${ }^{2}$ Il volume è

1 Una nota di Emilio Manzotti, a p. 71 della preziosa edizione commentata della Cognizione del dolore, (Torino, Einaudi, 1987) rileva la «contraddittoria [...] datazione».

2 Qui qualche ragguaglio bibliografico generale. Tra 1988 e 1993 esce l'edizione fondamentale delle Opere di Gadda diretta da Dante Isella per Garzanti nei "Libri della spiga": 5 volumi e 6 tomi. L'edizione include testi filologicamente accertati, con Note ai testi che per la prima volta danno le ricostruzioni storico-genetiche di ogni opera, mettendo a frutto le collazioni tra le stampe e gli anticipi in rivista, utilizzando gli 
aperto dal bellissimo Saggio introduttivo di Gianfranco Contini, e arricchito in chiusura da materiali d'autore: uno pseudo-dialogo AutoreEditore di stesura recente, dal titolo giocosamente pedante L'Editore chiede venia del recupero chiamando in causa l'Autore (che ragiona a mo' di autodifesa sulle ossessioni di Gonzalo), e la lirica Autunno recuperata dagli anni solariani. L'edizione einaudiana del '70 aggiunge due "tratti" inediti: l'VIII e il IX (databili intorno al 1938):3 Gadda è ai suoi ultimi anni, via via più sofferenti (come si è detto muore nel 1973, a Roma).

autografi quando già disponibili (come il prezioso Racconto italiano di ignoto del novecento), e i carteggi (anche quello allora inedito a Garzanti). Per la Cognizione, si rinvia inoltre alla fondamentale edizione commentata a cura di Emilio Manzotti: Torino, Einaudi, 1987, già cit., con molti materiali inediti estratti dagli archivi (citerò i riscontri testuali da questa edizione, anche per i riscontri al commento). Ma si vedano anche il testo e la Nota ai testi sempre a sua cura nel vol. I delle Opere Garzanti, cit., vol. I (1988), cui rinvio per la storia e il significato del testo nella carriera di Gadda. Sempre a Manzotti si devono pagine critiche di grande rilievo: il profilo sulla Cognizione in Letteratura italiana. Le Opere, diretta da A. Asor Rosa, IV. Il Novecento, t. II, Torino, Einaudi, 1996, pp. 202-337; e il capitolo gaddiano della Storia della letteratura italiana diretta da Enrico Malato, vol. IX, Il Novecento, Roma, Salerno, 2000, pp. 611-687. Manzotti ha edito inoltre sui «Quaderni dell'Ingegnere» einaudiani creati da Isella (2004, 3, 5-31) stesure precedenti dei tratti V-VI: La cognizione del dolore. Parte seconda. Le carte della Cognizione dei Fondi Roscioni e Citati (ora alla Biblioteca Trivulziana di Milano), cui si aggiungono i materiali del Fondo Liberati di Villafranca, non sono state ancora integralmente studiate. La prima già ricca ricostruzione delle vicende testuali della Cognizione risale a Roscioni, nella Nota introduttiva non firmata dell'ed. Einaudi 1971. Esce nel 2017, a cura di Paola Italia, Giorgio Pinotti, Claudio Vela, l'ed. per Adelphi della Cognizione, che presenta notevoli documenti familiari inediti (sulla costruzione della casa, sui testamenti, sulla vicenda dell'ipoteca, sui vari servitori di Longone e dintorni, sulla vicenda della vendita) attinti in particolare dal Fondo Liberati. Un riesame complessivo della situazione filologica della Cognizione sulle carte del Fondo Gadda nella Biblioteca Trivulziana, con nuove attendibili indicazioni sulla composizione del romanzo e delle sue parti, si legge ora in un denso saggio di Chiara Ornago, giovanissima allieva pavese, «La cognizione del dolore»: nuove indagini filologiche e ipotesi genetiche, «Strumenti critici», 150, 2019, 2, maggio-agosto, 221-240.

3 I "tratti" aggiuntivi dell'ed. '70 erano stati attribuiti autorevolmente al 1941 da Giancarlo Roscioni, che (come dirò oltre) soccorse in modo decisivo Gadda per il varo del volume einaudiano del ' 63 , e che ebbe l'agio di conoscerne le carte. Il '41 fu assunto dunque come dato certo, sino a quando Manzotti ne fece una cauta ma opportuna smentita (nell'ed. Garzanti del 1988, cit., p. 878), avendo ricavato da elementi delle carte autografe la retrodatazione al 1938 (per i dettagli in merito rinvio al saggio di Chiara Ornago, cit., pp. 223-224). 
Può sorprendere - ma nel contempo illumina le bizzarrie di Gadda - che dal '41 il capolavoro incompiuto sonnecchi quasi per un ventennio tra le pagine di una rivista. Vero è che subito, nel gennaio 1938, prima che il romanzo si affacci in rivista, Gadda pensa già di farne un libro, con uno slancio che a breve si rivela non realistico evidentemente perché il lavoro gli dà più filo da torcere di quanto ipotizzasse all'origine. ${ }^{4} \mathrm{Da}$ un certo momento in avanti, dopo l'uscita in rivista, Gadda propone il testo per il volume e vari editori lo sollecitano con insistenza. ${ }^{5} \mathrm{Ma}$ pur promettendo, anzi impelagandosi in più promesse, Gadda si decide tardi al libro, e solo perché, va detto, per contrastarne i ritardi cronici e le nevrotiche esitazioni, Einaudi ha la buona idea di mettergli alle costole un giovane di talento: Giancarlo Roscioni, poi suo grande studioso. Del resto, per pubblicare in volume nel '57 il Pasticciaccio riesumandolo dalle puntate di «Letteratura» (1946), già Garzanti gli aveva affiancato un giovane colto e brillante come Pietro Citati.

4 Un annuncio pubblicitario di «Letteratura» del 1938 (n. 5, gennaio-marzo; replicato nel n. 6, aprile-giugno) promette l'opera in volume per le edizioni della rivista (Fratelli Parenti). L'annuncio include una pittoresca sintesi d'autore (non firmata) con rinvio al Macbeth: riportata da Roscioni nella Nota introduttiva anonima dell'ed. 1971. Nel '38 stesso, n. 8, l'annuncio è però variato, poiché il volume promette di includere, oltre alla Cognizione, anche San Giorgio in casa Brocchi e Le meraviglie d'Italia, quest'ultimo effettivamente edito a sé nel '39. Nello stesso periodo Gadda enuncia il progetto per lettera ad alcuni degli amici più stretti (Bonaventura Tecchi, il cugino Gadda Conti). Cfr. su ciò: Italia, Pinotti, Vela nell'ed. Adelphi 2017, cit., pp. 275-278; e già, con dati lacunosi, Manzotti nell'ed. Garzanti 1988, Opere, I, cit., pp. 856-858. Interessante che il primo annuncio in «Letteratura» come le confidenze agli amici precorrano l'apparizione della Cognizione in rivista, dove comincia a essere stampata dal n. 7, luglio-settembre 1938. Si veda anche quanto scrive Liliana Orlando nella Nota al testo alle Meraviglie d'Italia (in C.E. Gadda, Opere, III, 1991, pp. 1238-1245), sul progetto iniziale di radunare anche la Cognizione nel volume che viene limitato presto (1939) alle sole Meraviglie, con dedica significativa alla memoria della madre (scomparsa nel'36).

5 Gadda propone La cognizione a Sansoni già nel '41, per poi acconsentire dopo un decennio, nel '52, a una richiesta di Vittorini per i "Gettoni" einaudiani, rincalzata dalle parole di Giulio Einaudi (sugli ingarbugliati contatti con più editori, cfr. Manzotti nell'ed. Garzanti 1988, cit., p. 866, e pp. 868-873; e Italia, Pinotti, Vela nell'ed. Adelphi, cit., pp. 307-308, 312-316). 


\section{UNA SISTEMATICA «COMPLESSITÀ»}

Tutto in Gadda parla di complessità. Ma a che cosa si deve questa sistematica «complessità», e come la si può descrivere? E soprattutto perché evocarla da parte nostra come un grimaldello non generico in grado di schiudere alcuni dei meccanismi dei testi e dell'universo gaddiano? Cerco di chiarirlo per sommi capi (chiedendo scusa delle semplificazioni).

Intanto, la «complessità» gaddiana riguarda sia il rovello dei nuclei tematici sia i compositi modi stilistici e linguistici e le prospettive del racconto, coinvolgendo lo stesso problema dell'incompiutezza. Compendiando in breve i molti elementi fortemente contrastivi della sua ardua scrittura, citiamo i ricorsi frequenti e profondi alla classicità: lessicali, ma anche sintattici, e anche immaginativi, attraverso metafore, allusioni, rinvii, il tutto incrociato spesso, con spregiudicato esercizio contaminatorio, a moduli di stile basso e popolare. Altrettanta passione Gadda dedica all'area dialettale: voci e locuzioni milanesi-lombarde, ma pure romanesche e napoletane. Terreno costante di prelievi è anche il linguaggio tecnico-scientifico, di cui per formazione l'ingegnere politecnico è molto esperto. Costrutti illustri di sintassi e lessico elevato convivono dunque con tratti forti del parlato (anche sintattici), con imprecazioni e parole oscene, e con affioramenti tecnici anche protratti. Per le caratteristiche mescolanze, il grande filologo e critico Gianfranco Contini, estimatore fedele e geniale di Gadda (straordinario il loro carteggio), ${ }^{6}$ ha parlato di espressionismo, risalendo audacemente per li rami sino a Dante lungo l'asse secolare della tradizione comica della nostra letteratura (sempre o spesso implicata con il dialetto), e risuscitando anche in particolare la vecchia e florida linea macaronica quattrocinquecentesca di area padana.

Per cogliere specificità e genesi della «complessità» di Gadda, è d'obbligo arretrare al Racconto italiano di ignoto del novecento, cantiere di romanzo incompiuto del 1924-'25, dove alle pagine narrative (che Gadda chiama Studî) si alternano sostanziosi appunti critici (nominati

6 Cfr. Gianfranco Contini-Carlo Emilio Gadda, Carteggio 1934-1963, a cura di Dante Isella, Gianfranco Contini e Giulio Ungarelli, Milano, Garzanti, 2009. 
Note) concentrati sugli aspetti tecnici del romanzo. ${ }^{7}$ Il Racconto italiano fu elaborato quando il giovane ingegnere, reduce da un'esperienza di lavoro in Argentina (1922-'24), approfittò del temporaneo agio economico per sospendere provvisoriamente il lavoro tecnico, dedicarsi alla laboriosa stesura del primo impegnativo romanzo e seguire i corsi di Filosofia all'Accademia scientifico-letteraria (ricavando poi dagli anni argentini, come è noto, il futuro travestimento sudamericano della Cognizione). La concezione della «complessità» matura dunque negli studi filosofici, concentrati in particolare, per la tesi (con Pietro Martinetti, stesa ma mai portata a termine), ${ }^{8}$ sulla teoria della conoscenza in Leibniz. La «complessità» in tale accezione, nel sentimento prevalente di una molteplicità non componibile, invade prima il Racconto italiano e poi è analizzata nello scritto "filosofico" Meditazione milanese steso nel 1928 ma pubblicato postumo da Roscioni che lo estrasse dalle carte dell'autore.' Qui Gadda riflette su problemi a lui sempre presenti: il conflitto tra bene e male, tra abnormità e norma (temi-chiave del Racconto italiano e di molta sua scrittura), i limiti della conoscenza e degli sforzi esplicativi, evocando di continuo la «prospettiva della complessità», l'intreccio mobile di relazioni, combinazioni, differenziazioni, divergenze, di punti di vista diversamente

7 Il Racconto italiano di ignoto del novecento fu pubblicato in edizione critica esemplare da Dante Isella, Torino, Einaudi, 1983 (da dove si cita), e da lui riedito nel vol. V* delle Opere, cit., Scritti vari e postumi, senza gli apparati variantistici.

8 I materiali della tesi, del 1929, dal titolo La teoria della conoscenza nei «Nuovi saggi» di Leibniz (contenuta in un quaderno del Fondo Roscioni), sono stati editi da Riccardo Stracuzzi nella rivista fondata da Dante Isella, I Quaderni dell'Ingegnere (d'ora in avanti QI), 4 (2006), pp. 5-38. Per moltissimi dati sugli anni giovanili e sui rapporti familiari, si veda la ricostruzione di Giancarlo Roscioni, Il duca di Sant'Aquila, Milano, Mondadori, 1997, pp. 246-247, e 253-254. È imprescindibile come chiave d'accesso alla comprensione gaddiana il suo precedente volume, La disarmonia prestabilita, Torino, Einaudi, 1969 ( $1975^{2}$ con integrazioni), prima esplorazione della cultura filosofica di Gadda. Sulla formazione filosofica di Gadda, si segnala per la ricca documentazione il lavoro di Guido Lucchini, Gli studi filosofici di C.E. Gadda (1924-1929), in Per Carlo Emilio Gadda, «Strumenti critici», 9, 75 (1994), 223-245, che rileva nei lavori filosofici di Gadda una prospettiva soprattutto letteraria, e censisce e studia anche i quaderni con gli appunti dei corsi universitari presenti nell'Archivio Garzanti. Il saggio è leggibile online sul sito dell'EJGS-«Edinburgh Journal of Gadda Studies»: https://www.gadda.ed.ac.uk/Pages/ resources/archive/filosofia/lucchinistudifilosofici.php.

9 Roscioni pubblica la Meditazione milanese nel 1974, per Einaudi. L'opera entra poi, a cura di Paola Italia, in Opere, V*, 1993. 
orientati e perpetuamente mutevoli. Come scrive Roscioni, le «idee» dibattute nella Meditazione lasciano «traccia indelebile» nel lavoro gaddiano. Sul côté propriamente letterario, tali «idee» influenzano la narrazione sia infondendole una tensione conoscitiva costante, anche se avviluppata, sia facendo emergere su tutti i piani del trattamento narrativo una visione complessa: cioè - per dirla con Gadda - plurale, combinata, molteplice. L'acuta «coscienza della complessità» (Meditazione milanese, pp. 668, 842, 845, dell'ed. in Opere), o «sensazione della complessità» (p. 842 ), genera e/o rafforza il gusto gaddiano preesistente per anomalie e contaminazioni. L'intera esecuzione narrativa in Gadda è dominata - coerentemente, in tanta difformità - da serie di fenomeni riconducibili al pluri-prospettivismo.

Sulla resa letteraria della «complessità» si interroga con insistenza il Racconto italiano, alla ricerca dei modi idonei per dare vita a «un romanzo della pluralità»(p. 88), che si giovasse del «punto di vista "organizzatore" della rappresentazione complessa» (p. 87), e che contenesse anche un'«idea plurale o sociale» (p. 96). ${ }^{10}$

\section{TRATTAMENTO NARRATIVO E FENOMENI DELLA «COMPLESSITÀ»}

In direzione plurale va per cominciare la mescolanza di generi, registri, stili, già in parte enunciata dall'autore nel Racconto italiano, ${ }^{11} \mathrm{e}$ poi analizzata nei notevolissimi saggi della "Prima parte" della raccolta garzantiana I Viaggi la morte, 1958 (datati 1929-1957, l'ultimo sul Pasticciaccio). Sugli azzardi contaminatori eccelle il denso Fatto personale... o quasi ${ }^{12}$ una dichiarazione di poetica e un'illustrazione genealogi-

10 Qualche altro riscontro dal Racconto italiano: «la combinazione, l'istinto della combinazione è nell'universo», p. 27; «essendo il mio un romanzo della pluralità», p. 88 ; «relatività dei momenti, polarità della conoscenza, nessun momento è assoluto», $i b$; «leit-motif della pluralità sul singolo», p. 273.

11 Con tanto rilievo che occupa una delle primissime note (24 marzo 1924), riservata a delineare le «cinque maniere» della propria scrittura (p. 14).

12 Del 1947, ma sviluppato in volume con addendi anche artistici, tra cui proprio l'amato Caravaggio, idolo giovanile. Per la bibliografia e per l'iter variantistico dei saggi della "Prima parte", cfr. la nota ai Viaggi la morte, in Opere, III, a cura di chi scrive, pp. $1315-1322$. 
ca della propria «maccheronea», che - precisa Gadda - congiunge satira e malinconia. Il ricorso alla «maccheronea» è un palese omaggio a Gianfranco Contini, amico, mentore, complice, che sin dalla recensione al Castello di Udine allacciava Gadda alla linea macaronica della nostra tradizione. ${ }^{13}$ Scrive Gadda, mettendo in rilievo nel proprio lavoro contro l'accusa dell'«esercizio barocco» - la «gioia» dell'«immergersi nella comunità vivente delle anime», nel loro antico linguaggio, e nel «sentimento dei molti»:

Dire per maccheronea è dunque, talvolta, [...] un immergersi nella comunità vivente delle anime, un prevenirne o secondarne in pagina l'ingenito impulso a descrivere il (la volontà definitrice del) reale, per allegri segni. Tenui sfumature, sottili vincoli o precipitati trapassi, dalla satira alla maccheronea. Dalla malinconia alla maccheronea. (In un senso ampio ed alto, resultano maccheronici dopo che lirici) i grandi lombardi anticlassici (contro l'apparato rinascimentale) il Fossano, il Foppa, il Moretto, l'allucinante violenza del Caravaggio: i fiamminghi della descrizione, del catalogo: l'animismo folle d'un Bosch.

Dire per maccheronea è più tosto un deferire, che un reluttare al sentimento dei molti: è interpretare e vivere $[. .$.$] il meccanismo della$ fluente conoscenza, della descrizione e catalogazione dell'evento.

Maccheronea non è $[. .$.$] un esercizio barocco d'una prezioseggiante$ stramberia, ma desiderio e gioia del dipingere al di là della forma accettata e canonizzata dai bovi: è gioia dell'attingere agli strati autonomi

13 Nello scritto sul Castello di Udine del '34, Contini (allora appena ventiduenne, filologo romanzo e già appassionato critico militante) spiega Gadda anche alla luce della vivace produzione multilinguistica "maccheronica", caratterizzata da incroci tra dialetto, volgare e latino e praticata dalla fine del Quattrocento in area padana tra Padova, Mantova, Brescia (il vertice fu come è noto il Baldus di Teofilo Folengo). Il multilinguismo maccheronico è stato analizzato minutamente nelle sue varie specie da Cesare Segre in alcuni dei saggi di Lingua, stile e società (Milano, Feltrinelli, 1963), molto vicini alle linee di lavoro di Contini, suo "terzo maestro" (dopo Santorre Debenedetti e Benvenuto Terracini). ̇̇ posteriore e fecondissima, dagli anni ottanta in avanti, l'apertura di Segre alla lezione di Bachtin, geniale evocatore della tradizione della satira menippea, che porta per Segre almeno al bellissimo libro einaudiano del '91, Intrecci di voci, con investigazione diacronica e sincronica dei fenomeni polifonici nel Novecento. Si veda in particolare il saggio dell'85, Punto di vista, polifonia ed espressionismo nel romanzo italiano (1940-1970), ivi raccolto, che legge l'appena edito Racconto italiano con l'occhio agli studi di Bachtin su punto di vista, plurivocità, polifonia, sorprendentemente consonanti con alcune delle riflessioni narratologiche gaddiane. 
della rappresentazione, l'umore freatico delle genti, atellane o padane che le fossero, delle anime. ${ }^{14}$

La partecipe dichiarazione sulla comunità dei parlanti come fonte ispirativa del lavoro gaddiano consuona in profondità con l'Avvertenza iniziale che, con funzione di commento generale, precede le note del bellissimo "disegno" milanese Quattro figlie ebbe e ciascuna regina, inserito da ultimo nell'Adalgisa del 1944, e anticipato poco prima del volume su «Letteratura» (gennaio-marzo 1942). Siamo sempre negli anni quaranta, appena dopo la Cognizione e nei pressi dell'Adalgisa, anni evidentemente fervidi di riflessione su certi aspetti della scrittura, in particolare sul suo alimento linguistico popolare e corale. Ecco il testo dell'Avvertenza:

In questo disegno milanese «su cartone vecchio», alla tentata rappresentazione di un «interno» si sono voluti adibire, a tratti, i modi mentali e i modi idiomatici proprî de' personaggi che in quell'interno esagitavano il loro spirto vitale. L'orditura sintattica, le clausole prosodiche, l'impasto lessicale della discorsa, in più che un passaggio, devono perciò ritenersi funzioni mimetiche del clima, dell'aura di via Pasquirolo o del Pontaccio: che dico, dell'impetus e dello zefiro parlativo i quali dall'ambiente promanano, o prorompono. E ciò non soltanto nel dialogato, ma nella didascalia e nel contesto in genere, quasicché a propria volta l'autore si tuffi nella bagnarola e nell'acqua medesime ove poco prima erano a diguazzare i suoi colombi. Quanto all'impeto, è un impeto ricco, nativo, a cui non si sogliono imporre freni accademici o mediazioni teologiche: e vien celebrato nelle cronache quale «bella spontaneità» $\mathrm{o}$ «bella immediatezza del dire». E del ragionare e dell'essere, dunque. ${ }^{15}$

Nel quadro della ricerca del molteplice possiamo inscrivere senza forzature anche gli estri linguistici gaddiani, data la propensione dello scrittore a giovarsi di ciò che lui chiama tutte le «contaminazioni intol-

14 Cfr. C.E. Gadda, Opere, III, pp. 498-499.

15 Cfr. C.E. Gadda, Opere, I, pp. 374-375. L'Adalgisa è stata riedita a cura di Claudio Vela nelle edizioni Adelphi nel 2012 (la nota al testo è sorretta da importanti documenti prima non noti), dove il passo, invariato, si legge a p. 109. Sulla vicenda particolare di Quattro figlie ebbe..., rinvio a Luca Mazzocchi, Per «Quattro figlie ebbe e ciascuna regina» dell' «Adalgisa». Genesi e primo abbozzo inedito, in «Strumenti critici», 150, 1/2019, gennaio, 107-126. 
lerabili»: le varianti morfologico-fonetiche della parola, le variazioni sociali e temporali classificate poi dalla moderna sociolinguistica (diafasiche, diastratiche, diatopiche, ecc.). Si veda l'elogio edonistico del linguaggio e delle sue infinite possibilità contenuto in Lingua letteraria e lingua dell'uso (1942):

I doppioni li voglio, tutti, per mania di possesso e per cupidigia di ricchezze, e voglio anche i triploni, e i quadruploni [...]: e tutti i sinonimi, usati nelle loro variegate accezioni e sfumature, d'uso corrente, o d'uso raro rarissimo. Sicché dò palla nera alla proposta del sommo e venerato Alessandro, che vorrebbe nientedimeno potare, ecc. ecc.: per unificare e codificare: «d'entro le leggi, trassi il troppo e 'l vano». Non esistono il troppo né il vano, per una lingua. ${ }^{11}$

Proseguendo nel regesto del complesso, ne ricevono luce anche $\mathrm{i}$ caratteristici modi gaddiani di racconto, digressivi e antilineari. E lo stesso ricorrente gusto del catalogo e dell'elenco, strumento formidabile di rappresentazione dell'«eteroclito» straripante.

Si colloca su linea affine, sotto il segno di complicazione e inclusione di materiali disparati, la pratica delle note auto-esegetiche, congenita nell'officina gaddiana e fondata su impulsi umoristici di base. Ulteriore ricerca del deviante e del dettaglio, e insieme dell'interruzione, l'uso delle note si spinge a infrangere le sponde canonicamente testuali (tanto che Genette denomina il fenomeno «malattia delle note»). ${ }^{16}$ Alcuni caratteri in sintesi delle note gaddiane: nascono già nella scrittura privata del Giornale di guerra e di prigionia, esplicitando una vocazione originaria sia al chiarimento totalizzante sia alla diramazione; sono praticate in tutti i generi; vedono un acme creativo negli anni Trenta (nel 1934 Gadda legge decisivamente Swift); e conoscono una dedizione organizzata negli anni Quaranta. ${ }^{17}$ È significativo al riguardo che nel fervido cantiere satirico

16 Cfr. Gérard Genette, Seuils (Paris, Ed. Seuils, 1987), tradotto in Italia nel 1989, per Einaudi, con il titolo Soglie. I dintorni del testo.

17 Mi si permetta un rinvio a un mio studio, Sul sistema delle note in Gadda: lavori in corso, contenuto in (a cura di) Monica Marchi e Claudio Vela, Meraviglie di Gadda. Seminario di studi sulle carte dello scrittore, Pisa, Editore Pacini, 2014, pp. 115136. E al successivo, condiviso con Lorenzo Panizzi, La «malattia delle note» e altre bizzarrie. Gadda verso satira e Swift, in «Strumenti critici», 1/2015, gennaio, 85-118. 
dell'Adalgisa, edita per Le Monnier nel 1944 e dotata stabilmente di ben 277 note, la parte maggiore di esse sia scritta in vista del volume, anche grazie al favore di Giuseppe De Robertis, estimatore antico di Gadda e delle note stesse e direttore della collana ospitante. Nel fervore dell'Adalgisa appena edita rigurgitante di note e sempre prediligendo 1' "eccentrico" contro il canonico, Gadda è tentato dalla proposta dell'editore Nando Ballo di dedicarsi a un «libro di sole note», ${ }^{18}$ per cui Ballo suggerisce anche il bel titolo paradossista Variazioni senza tema. Gadda si lascia incuriosire e attrarre («Davvero lei mi suggerisce una idea che mi si attaglia»), riflette sulla possibile esecuzione strutturale, apprezza la «direzione essaystica a cui avevo l'animo già rivolto da anni». Ma il libro non si fa, conteso da altri progetti da completare.

\section{Sull'inCOMPIUTEZZA. Il CASO “AFFETTIVO” DELla «COGNIZIONE»}

Ed eccoci all'incompiutezza, che investe tutte le narrazioni gaddiane salvo alcuni racconti, e che pure si inscrive a buon diritto nel segno di una mutevole «complessità», tanto varia e precaria da rendere impossibile il chiudere. Si veda l'“autogiustificazione” in merito tratta dall'intervista con Moravia del '67:

Il rifiuto del finito, nel caso del giallo è dovuto al consapevole tentativo di chiudere in apocope drammatica il racconto che tendeva a deformarsi. Nel caso della Cognizione, l'incompiutezza ebbe moventi lirici, affettivi, che contrastano il canone estetico e strutturale della narrativa pura, la quale, secondo me, non esiste, come non esiste la strategia pura. ${ }^{19}$

Che Gadda ne parli nel '67, avendo edito in volume i suoi due grandi romanzi entrambi inconclusi: il Pasticciaccio, dieci anni prima, e

18 Cfr. C.E. Gadda, Lettere agli editori Rosa e Ballo (1943-1946), a cura di Dante Isella, QI, 5, 2007, 47-60. Le lettere cit. sono alle pp. 54-55.

19 In Carlo Emilio Gadda, «Per favore, mi lasci nell'ombra». Interviste 19501972, a cura di Claudio Vela, Milano, Adelphi, 1993. 
nel '63 La Cognizione; e che ne parli per di più con Moravia, narratore per così dire al quadrato, sbilanciandosi con termini drastici a lui non abituali («la narrativa pura [...], secondo me, non esiste») è doppiamente notevole. La pur seducente definizione dell'incompiutezza come «tentativo di chiudere in apocope drammatica» sembra voler fugare con qualche nervosismo ogni dubbio come artisticamente inessenziale. $\mathrm{Va}$ ovviamente osservato che per La cognizione vengono chiamati in causa i particolari «moventi lirici, affettivi» in quanto responsabili di intralciare la narrazione «pura», data peraltro come inesistente.

$\mathrm{Ma}$, guardando al fenomeno dalla parte dei lettori e critici che vogliono capirne di più, che significato dare alla permanente incompiutezza? Se, come si diceva, le istanze generali di «complessità» e pluralità comportano ovviamente riluttanza a chiudere, e difficoltà a individuare una soluzione, per La cognizione vale altrettanto, o di più, l'elemento emotivo interno, prospettato da Gadda nella citazione stessa (e messo in rilievo da Contini nel Saggio introduttivo): il veto profondo che qui blocca la conclusione ha a che fare con la storia personale dell'autore, con tanta oscura potenza da essere implicato con il grave fantasma del matricidio che il testo suscita e insieme non esegue. Matricidio già tentato, come ben si sa, in un racconto incompiuto del 1928, Dejanira Classis (come lo rinomina Isella dagli autografi) o Novella seconda (come fu chiamato nell'edizione del 1971 per Garzanti). La novella («seconda» nella terminologia gaddiana perché seguiva La Madonna dei filosofi $)^{20}$ si ispirava alla cronaca milanese coeva, attestata sui giornali (il delitto Pettine): il caso di un giovane, reo confesso dell'uccisione atroce della madre, i cui pezzi di cadavere nascose nell'armadio per giorni. La narrazione lì fu interrotta alla preistoria, fermandosi alla lenta ricostruzione della giovinezza della donna. Ma i materiali preparatori chiariscono i densi retroscena psicologici: tra le carte Gadda si interroga, un decennio prima della Cognizione, sul possibile colpevole del matricidio, scagionando il figlio (come farà poi anche per La cognizione nei materiali compositivi conservati). ${ }^{21}$ Non senza insinuare già la percezione $\mathrm{o}$

20 Per dettagli su questa serie di Novelle ("prima", "seconda", "terza"), cfr. ora Francesco Venturi, Da «Novella $1^{a} \gg$ a $\ll$ Novella $3^{a} »$ : percorsi sommersi della narrativa di Gadda tra gli anni'20 e'30, «Strumenti critici», 152, II, 2020, maggio-agosto.

21 Così Gadda scrive per Renzo Pettine tra le carte preparatorie: «Il ragazzo 
il sospetto da parte della madre che l'uccisore fosse il figlio (ancora come accadrà nella Cognizione).

Non c'è dubbio che i tormenti della Cognizione piombino abbastanza nudamente dai recessi della storia familiare di Gadda. Il presunto formalista Contini capì molto bene che lo stile gaddiano era alimentato dalla nevrosi personale fin dal suo primo giovanile intervento senza alcuna conoscenza diretta dell'autore (sul Castello di Udine, 1934). E nel 1963 il Saggio introduttivo alla Cognizione è tanto lucido e penetrante nel sondare le nevrosi di Gonzalo-alter ego di Carlo Emilio (Contini parla di un' «arte macaronica esercitata su una materia [...] freudiana») da allarmare sino al panico l'autore, che legge in anteprima le splendide ma scottanti pagine. Così appare da due lettere a Contini del '63, gentilissime ma alterate, dove si esprime un grave turbamento per i riferimenti personali, e si arriva a suggerire ipotesi di emendamenti, si dica pure ininfluenti e insensati. ${ }^{22}$ Si ricordi che Contini proprio in apertura compara Gonzalo alla proustiana e omosessuale Mademoiselle de Vinteuil, grazie all'indizio rivelatore di un gesto comune ai personaggi: l'oltraggio al ritratto paterno. Nelle righe subito successive, il «sentimento dominante» del protagonista è individuato con sicurezza nell'«astio verso la famiglia», nel «trauma infantile da carenza d'affetto». Non solo, ma vengono giudicate «futili» e pretestuose le ragioni di rancore familiare addotte da Gonzalo a sua difesa: e cioè gli sperperi genitoriali per costruire la «fottutissima casa» di Longone, i soldi devoluti per le campane della chiesa del paese, l'«indulgenza» della madre verso i servitori e verso i ragazzini del paese («somarelli» accuditi con generose ripetizioni): tutte attenzioni - secondo il punto di vista sofferente di Gonzalo - sottrattive dell'amore materno.

Sta di fatto, venendo ancora all'incompiutezza, che il turbamento

sciagurato, corrotto, vittima non ha ucciso la madre, ma ha solo presenziato, nell'impossibilità di aiutarla, alla uccisione di lei. La scena terribile lo ha reso demente» (riportato da Isella in Opere, II, p. 1318).

22 Cfr. Contini-Gadda, Carteggio, cit., pp. 189-191. Questa, ripresa per stralci, l'annotazione che qui fa Contini alle dolorose lettere: «Episodio che sfiora la paranoia quello provocato dalla mia introduzione alla Cognizione del dolore in volume [...]. Le difficoltà mi furono illustrate da Roscioni [...]», pp. 190-191. L'angoscia dell'autore poté sanarsi con minime correzioni marginali, che lasciarono del tutto intatta la sostanza testuale. 
affettivo verso la famiglia e verso la madre è tale che mai Gadda si arrischia a portare all'estremo La cognizione, e tanto meno riapre il problema quando la raccoglie in volume nel 1963. Oltre l'acme del terribile delitto, gravato dai sospetti che si sono detti, Gadda si sottrae tenacemente all'epilogo. Sono continui nel racconto gli indizi disseminati contro Gonzalo - sospettato dai servi, che ne danno foschi ritratti, e dalla stessa madre - preparati con cura nei materiali compositivi, ma la vicenda non si può concludere, troncandosi appunto in «apocope drammatica». Il delitto c'è, descritto impietosamente nelle scene finali come già in «Letteratura». Ma i colpevoli sono più di uno, nelle varie ipotesi che le carte consegnano. ${ }^{23}$

\section{5. «LA COGNIZIONE»: IL «FULMINE», LA MORTE DELLA MADRE, IL LUTTO}

Per lo svelamento delle implicazioni affettive la cronologia degli eventi privati e di quanto si sa del lavoro ai testi è illuminante. La morte materna avviene il 2 aprile 1936. Gadda ha allora in corso da ben cinque anni il romanzo satirico Un fulmine sul $220,{ }^{24}$ entrato in scena subito come un attacco radicale e furente contro la ricca borghesia milanese («al di là c'è il manicomio, subito», confida nel '34 nel pieno del lavoro

23 Ricostruisce la vicenda Giancarlo Roscioni, alla luce di alcuni frammenti inediti e materiali preparatori del romanzo, richiamando anche il risvolto della princeps ispirato dall'autore: cfr. La conclusione della «Cognizione del dolore», aggiunto da Roscioni nella riedizione della Disarmonia prestabilita (Torino, Einaudi, 1974²), pp. 161 174. Roscioni ricorda (p.162) che nel risvolto del 1963 si legge che la madre subì l'aggressione «delle guardie della "vigilancia para la noche" [...] mentre atroce le si insinuava nell'animo il sospetto che ad organizzare l'aggressione fosse stato proprio il "tristo" figlio Gonzalo».

24 Edito pure magistralmente da Dante Isella nel 2000: cfr. Carlo Emilio Gadda, Un fulmine sul 220, Milano, Garzanti, 2000. Le fasi complesse del lavoro gaddiano sono ricostruite puntualmente dal gran disordine dei materiali nella Nota al testo, pp. 375 303: ideato nel 1931, il Fulmine conosce una prima fase nel '32; una seconda tra '33-'34; una terza, molto parziale, nel '36. Isella anticipò una parte significativa del Fulmine con apparato di varianti nella raccolta gaddiana Disegni milanesi (Pistoia, Can Bianco, 1995), che, oltre al Fulmine a sua cura, presentava San Giorgio in casa Brocchi, a cura di G. Pinotti, e L'incendio di via Keplero, a cura di P. Italia. 
all'amico di giovinezza Ambrogio Gobbi). ${ }^{25}$ Il Fulmine è ripreso tra il giugno '35 e nel '36 (purtroppo non è testimoniato il mese) nel tentativo di una ridisegnazione complessiva, in verità tutt'altro che chiara. ${ }^{26} \mathrm{Il}$ Fulmine è forse in crisi per più ragioni convergenti: la difficoltà di saldarne i molti materiali accumulati in più stesure, e di gestirne l'intreccio; il finale tragico della morte dei due amanti abbozzato in un solo e scarno documento superstite; l'espansione esuberante della vitalissima Adalgisa, che erode lo spazio dell'esangue Elsa. Nel 35, dopo un'interruzione abbiamo citato i segnali di una ripresa inventiva, non perspicua, ma che certo cambia l'assetto con nuovi inserti.

Il lutto del 3 aprile interviene potentemente a modificare le cose anche sul piano della scrittura. Nelle lettere agli amici più stretti del 1936-1937, Gadda esprime per la morte della madre un'angoscia di colpa fortissima. ${ }^{27}$ In particolare, così scrive al cugino Piero Gadda Conti (dicembre 1936), anche prefigurando passaggi della Cognizione, come il «Vagava, sola, nella casa» e i rabbiosi alterchi:

Ora che le ributtanti pratiche che seguono la scomparsa di una persona cara sono ultimate: municipio, pretore, notaio, ecc., l'imagine di

25 In una lettera del 7 aprile raccolta tra le Lettere agli amici milanesi, a cura di Emma Sassi, Milano, Il Saggiatore, 1983. pp. 45-48. Così del Fulmine: «Adesso sto lavorando a una novella del medesimo tipo [di San Giorgio in casa Brocchi], ma più lunga [...]. Essa risente del mio stato d'animo ed è condotta sui limiti estremi dell'acredine: al di là c'è il manicomio, subito», p. 47.

26 Il progetto di riorganizzare l'insieme, anche con parti di nuova stesura, risale al giugno '35. Gadda stese un nuovo piano complessivo datato 5 giugno 1935 (è il documento riportato nella Nota al testo, p. 324), piano frammentato e rapido, non ben individuabile, tanto che Isella parla di un riassunto tracciato in «modi enigmaticamente allusivi», p. 301. Del '36, purtroppo senza precisazione del mese, è il capitolo appena abbozzato che avrebbe dovuto costituire il nuovo inizio, Vita difficile nei prati di San Colombano: cfr. ed. Isella, pp. 213-233.

27 A Contini (nel Carteggio, cit., p. 107); al cugino Piero Gadda Conti (lettere raccolte in parte e commentate in Piero Gadda Conti, Le confessioni di Carlo Emilio Gadda, Milano, Pan, 1974); a Tecchi (cfr. Carlo Emilio Gadda, A un amico fraterno. Lettere a Bonaventura Tecchi, a cura di Marcello Carlino, Milano, Garzanti, 1984). Si veda anche a Lucia Rodocanachi, in Carlo Emilio Gadda, Lettere una gentile signora, a cura di Giuseppe Marcenaro, con un saggio di Giuseppe Pontiggia, Milano, Adelphi, 1983, dove si legge un accorato dolore nella lettera del 31 maggio 1936: «ho avuto mesi tristissimi, anche per la morte di mia Madre, anzi soprattutto per questo», p. 53. 
Lei vecchia e senza aiuti mi ritorna e oltre tutto un indescrivibile rimorso mi prende per i miei scatti, così inutili e così vili. Io ho troppo sofferto e certo non ero padrone di me, ma ciò non toglie che la mia angoscia sia ora grandissima. ${ }^{28}$

E all'«amico fraterno» Tecchi, 16 gennaio 1938:

Il mio animo è sempre molto abbattuto dalla ambascia per la morte di mia Madre e dai rimorsi che ho per il mio contegno verso di lei negli ultimi anni - rimorsi che talora mi appaiono insopportabili. ${ }^{29}$

Il lutto materno e la depressione allontanano Gadda, attanagliato da rimorsi e sensi di colpa, dalla satira beffarda del Fulmine, troppo aggressiva contro la propria città, contro la propria classe, contro la propria tribù familiare, e lo trascinano su altri temi e "trattamenti". Il "romanzo familiare" della Cognizione esplode per così dire dal trauma.

L'impegno inventivo-emotivo si sposta quasi per necessità dal Fulmine sul nuovo racconto cominciato nei «primi mesi $1937 »,{ }^{30}$ che investiga con inaudito ardire il legame spinoso con la madre. Aggrovigliata con il «dolore» messo con evidenza a titolo, la Cognizione, avviata d'impulso, è invasa anche dalle crudezze satiriche proprie di Gadda e persino da violenta comicità. Ma, dopo le stentate approssimazioni giovanili, la morte materna spinge lo scrittore ad affrontare l'oscura relazione e le esacerbate ferite infantili, facendo riemergere cupe fantasie parallele che da parecchio gli giravano in testa: il rovello di Amleto che lo inseguiva dalla giovinezza (la vendetta sulla madre a risarcimento dell'uccisione paterna); la "colpa condivisa" di matrice dostoewskiana (discendente dall'analisi freudiana dei Fratelli Karamazow); il matricidio, in qualche modo desiderato (come nei Karamazow lo fu l'uccisione del padre). La storia è calata con ottima intuizione nella gran mascheratura sudamericana, che ne consente un'esecuzione distanziata e umoristica. Le puntuali indagini filologiche di Chiara Ornago legittimano l'ipotesi che il nucleo originario di scrittura

28 Cfr. Piero Gadda Conti, Confessioni, cit., p. 42.

29 Cfr. Carlo Emilio Gadda, A un amico fraterno, cit., pp. 126-127.

30 Così si legge negli autografi. Si veda ora Chiara Ornago, cit., p. 225. 
sia proprio quello materno, della figura fragile e senile che «vagava sola per la casa».

Tutto si salda con combinazione urgente di ragioni emotive e artistiche: il lutto materno, l'interruzione del Fulmine ('36), l'avvio della Cognizione («primi mesi 1937»). Da segnare, nel maggio del '36, in una confidenza a Contini una prima ripresa del lavoro letterario:

Ho fatto qualche articolo sull'«Ambrosiano»: solo in questi giorni riprendo un po' a lavorare. Fra l'altro la mia casa di campagna (bella grana anche questa) mi procura più grattacapi che una suocera isterica. [...] Mi vendicherò. ${ }^{31}$

Contini commenta lapidariamente il finale «Mi vendicherò»: «è il primo germe della Cognizione». Nel novembre del'37 Gadda provvede con affanno alla vendita della casa di Longone, profondamente connessa con l'infanzia e i rovelli familiari.

Appena fuori dal lavoro accanito alla Cognizione, affidata per tormentose puntate alla rivista, sotto il pungolo amichevole ma costante di Alessandro Bonsanti, Gadda pensa al geniale reimpiego del Fulmine nei racconti dell'Adalgisa, con il trionfo umoristico delle note, quasi tutte aggiunte per il volume Le Monnier (1944).

\section{OSSESSIONI E FURORI IN GONZALO}

Nella coscienza lacerata di Gonzalo agiscono ossessioni e furori che colpiscono la madre, o, quando rivolti all'esterno come frequentemente succede, si generano dal rabbioso rapporto verso la madre. La tenerezza verso la madre è inghiottita e rovesciata in invettive incontenibili. Un esempio, efficacissimo, di repentino ribaltamento amore-odio:

Avrebbe voluto inginocchiarsi e dirle: "perdonami! perdonami! Mamma, sono io!". Disse: "Se ti trovo ancora una volta nel braco dei maiali, scannerò te e loro....” pp. 436-37. ${ }^{32}$

31 Da Contini-Gadda, Carteggio, cit, p. 108. La lettera è del 26 maggio.

32 Il «braco dei maiali» è ripresa dantesca, Inferno, VIII, 50 (canto degli iracondi, 
In Gonzalo, come in Carlo Emilio, Roscioni rileva, concordemente con Contini, ciò che nel Duca di Sant'Aquila chiama incisivamente la sindrome del «mal aimé», evidente nel tema virgiliano del figlio infelice «cui non risere parentes ${ }^{33}$ che nutre il romanzo e che si reperisce in un bellissimo frammento non entrato a testo, presente nell'ed. Manzotti da cui stralcio:

La sua vita non aveva conosciuto stagione: non primavera, sotto la ferula della miseria e del sadismo materno: non estate, né dolce autunno, né carità di figli, né nulla $[\ldots]$

2004 La madre avrebbe dovuto strozzarlo dopo otto giorni, se avesse avuto la pietà e le rettitudine della pantera. Invece lo aveva allattato, allevato, educato: educato, soprattutto educato! (p. 527, p. 532)..$^{34}$

Il «mal aimé» Gonzalo si sente percepito come «prova difettiva di natura»; ed è significativo che la pesante diagnosi non sia espressa dal protagonista, bensì serpeggi tra i pensieri della madre e sia messa a cari-

significativamente) «che qui staranno come porci in brago». Gadda usa il sintagma anche in un passo dell'iperletterario e rabbioso Tendo al mio fine, proemio del Castello di Udine: «Sarò il poeta del bene e della virtù, e il famiglio dell'ideale: ma farò sentirvi grugnire il porco nel braco» (Opere, I, p. 119). Nella tesi triennale su Gadda e sulla satira classica menippea, un allievo pavese, Luca Mazzocchi, ha segnalato un riscontro precoce nelle Lettere agli amici milanesi, cit, che riporto: «ò finto de non vedello quanno magnava la minestra cor cortello per annaspà i cavoli che ce stanno e lo riporto, de non sentillo quando sbrodolava come "u porco ner braco» (dalla lettera all'amico Ambrogio Gobbi, 21 gennaio 1916), in un pure precoce ma non insolito romanesco. Già nel Giornale di guerra e prigionia, Gadda scriveva sulla madre, passando dall'osservazione rispettosa all'esplosione d'ira: «Tanti sacrifici fa: e tanti si assume volontariamente per esser troppo buona coi porci fottuti», ora in Opere, IV, p. 645 .

33 Il tema del «cui non risere parentes» è discusso lungamente in chiave ironica da Gadda anche in Psicanalisi e letteratura (datato 1946, edito nel 1949 in rivista, «La Rassegna d'Italia», infine raccolto nel volume saggistico I viaggi la morte, 1958, ora in Opere III, cit., pp. 459-462).

34 Sull'educazione trattata con feroce satira da Gadda, richiamo per affinità le esilaranti riprese e buffonesche dilatazioni à bâton rompus del compianto e grande Alberto Arbasino nei Fratelli d'Italia, specie nel capitolo La condizione del dolore, omaggio gaddiano sui generis, aggiunto ex novo nell'edizione ultima, 1993. (Un rapido rinvio a Clelia Martignoni, Elisabetta Cammarata, Cinzia Lucchelli, La scrittura infinita di Alberto Arbasino, Novara, Interlinea, 1999). 
co di lei, erogatrice avara d'amore..$^{35}$ Per questa via "sovrana", la diagnosi è per Gonzalo una condanna inappellabile.

Questa è dunque, scavando all'osso, l'insanabile, l'affannosa «cognizione del dolore»??6 Il titolo, bellissimo e misterioso, batte sul cuore dell'opera: la «cognizione del dolore». «Cognizione» osservata e raffigurata in chi? Nei due protagonisti, infelici e incomunicanti?, la signora e il figlio, ognuno per la propria dolorosa parte? $\mathrm{O}$ accreditata al solo Gonzalo, colpito dal «male invisibile»?, che pure la madre dolorosamente coglie $(\ll \mathrm{Da}$ anni aveva intuito, di suo figlio. La povera madre aveva lentamente compreso»). ${ }^{37}$

Alla «cognizione» fa da contrappunto e rovesciamento il «delirio», altra parola-chiave del testo, relativa a Gonzalo e associata precisamente dal narratore a patologie psichiche: «Forse il suo - commenta il narratore - era quello che Sérieux, Capgras, e altri psichiatri contemporanei, hanno efficacemente chiamato "delirio interpretativo" $\gg .^{38}$ L'opposizione-oscillazione tra «cognizione» e «delirio» palesa il franto universo emotivo-cognitivo rappresentato nel romanzo.

\section{Altri SEGNALI DI COMPLESSITÀ: LA RIDDA DI VOCI NEL ROMANZO}

Nella Cognizione, come nell'Adalgisa, e come nel Pasticciaccio, il

35 «Sì: c'era il suo figlio [...]. Il primo suo figlio. Quello nel di cui corpicino, oh! giorni!, aveva voluto vedere la prova difettiva di natura, un fallito sperimento delle viscere dopo la frode accolta del seme», pp. 271-272. Commentando la «prova difettiva», Manzotti aggiunge in nota (p. 271) riscontri precisi con altri testi gaddiani (da Eros e Priapo; dai Viaggi di Gulliver, cioè del Gaddus; dai Viaggi la morte). Si veda anche l'analisi (con riscontri su documenti inediti) di Roscioni, Il duca di Sant'Aquila, cit., p. 53; e Elio Gioanola, Topazi e alte gioie familiari, Milano, Jaca Book, 2014, p. 240.

36 La cognizione, pp. 271-72.

37 La cognizione, p. 311.

38 Il «delirio interpretativo» con rinvio agli psichiatri Sérieux e Capgras è evocato da parte del narratore nel colloquio Gonzalo-dottore per spiegare le alterazioni di Gonzalo (cfr. pp. 210-211, e nota relativa di Manzotti). Interessante invece che nello pseudo-dialogo Editore-Autore del 1963 (come già rileva Manzotti nel commento) un Gadda iper-turbato respinga per Gonzalo l'ipotesi del «delirio interpretativo», e addossi l'«ossessione» del protagonista alla responsabilità degli «altri»: «La ossessione di Gonzalo [...] procede dagli altrui errori di giudizio e dalle altrui, singole o collettive, carenze di contegno sociale» (pp. 489-490). Palesemente è una rilettura tarda d'autore in parte deviante dal testo originario. 
testo è sapientemente brulicante di tante voci, talora inestricabili, tra le quali ogni tanto emerge anche quella del narratore, in un vortice di prospettive alla cui decifrazione critica giovano gli studi di Bachtin (di un Bachtin ante litteram trapelante nel Racconto italiano ha parlato, come già di è detto, Cesare Segre). ${ }^{39}$

Sfruttando con eccellenza il ricorso alle voci e parole altrui, l'ingresso di Gonzalo in scena è ritardato ad arte. Il romanzo si avvia sul pittoresco esordio sudamericano (una trentina di pagine) raccontando il Maradagàl, reduce dalla guerra con il Parapagàl, e il Nistitúo. Il preambolo, se da un lato fornisce le fantasiose coordinate di spaziotempo-azione, funziona soprattutto come accesso satirico-umoristico alla grave «cognizione del dolore». Gonzalo, il truce protagonista, è anticipato in visione multipla e «complessa» attraverso le iperboliche parole altrui: ciò che i lukonesi pensano di lui (pp. 73-74), ciò che ne pensa il peone José (pp. 71-77), ciò che ne pensa la Peppa (p. 77), e ancora José e il coro dei lukonesi (pp. 73-83). Comico-iperbolichedeformanti tracce della sua fama accompagnano le divagazioni del dottor Higueroa, il medico condotto chiamato a visitarlo (a p. 71: «Al decimo giorno, il 28 di agosto, verso le undici della mattina»), durante la passeggiata a piedi per raggiungere la casa (pp. 83-99). Nelle battute della Battistina, incontrata per strada dal dottore sul far del mezzogiorno (pp. 116-128), entrano minacciosi segnali degli attacchi di Gonzalo contro la madre (indizi di possibile colpevolezza accumulati ad hoc dal narratore).

La sarabanda che conduce alla villa, alla famiglia Pirobutirro, a Gonzalo, introduce via via molti elementi del contesto: i reduci di guerra, i mutilati, il losco Manganones, la lavandaia Peppa (quasi in margine, sbuca per la prima volta «il figlio della Signora», p. 30; nonché la «Signora» della villa Pirobutirro, p. 32; e il muro di cinta facilmente violabile). Quindi irrompe la bellissima tirata sulle ville, ville!, villette; e seguono a rotta di collo le vicende della Villa Maria Giuseppina (già del Vate Carlos Caçoncellos, e poi dei Bertoloni), il colonnello Di Pascuale, e di qui il buon dottor Higueroa.

Il racconto è tanto accidentato e polifonicamente plurale, che

39 Cfr. supra, n. 12. 
talora è difficile individuare con sicurezza le voci in campo: chi racconti, di chi si riferiscano, sensazioni, ipotesi, pensieri, ricostruzioni. Così accade anche per l'attacco patemico-lirico dagli echi dannunziani sul leggendario «male invisibile» (che prosegue in nota con una pseudocitazione-pastiche da Bandinelli): $:^{40}$ certo non è riferibile al bonario dottore, tanto meno lo è ai lukonesi, né a Gonzalo, ma va attribuito a un narratore che si infiltra nelle pieghe del testo, a tratti umoristicamente, qui liricamente:

E c'era, per lui, il problema del male: la favola della malattia, la strana favola propalata dai conquistadores, cui fu dato raccogliere le moribonde parole dello Incas. Secondo cui la morte arriva per nulla, circonfusa di silenzio, come una tacita, ultima combinazione del pensiero.

È il «male invisibile», di cui narra Saverio López, nel capitolo estremo de' suoi Mirabilia Maragdagali.

Analoghe parole ritornano più avanti, a mo' di Leitmotiv, espresse stavolta dalla sapiente intuizione materna:

Da anni aveva intuito, di suo figlio. La povera madre aveva lentamente compreso [...]: Era il male oscuro di cui le storie e le leggi e le universe discipline delle gran cattedre persistono a dover ignorare le cause, i modi, e lo si porta dentro di sé per tutto il fulgurato scoscendere d'una vita, più greve ogni giorno, immedicato. Forse il «male invisibile» di cui parla Saverio López: dettogli da moribonde parole dello Incas: e ne dice, con licenza de' superiori, al capitolo estremo de' suoi Mirabilia Maragdagali. (pp. 311-12)

\section{IL CARDINE DEL LEITMOTIV}

Riprese e Leitmotive di ispirazione latamente musicale stabiliscono nella Cognizione «complesse» connessioni melodico-ritmico-semantiche, con più funzioni anche percepibili in contemporanea. Ancora è necessario tornare al fondamentale banco di prova che sono le riflessioni del Racconto italiano, dove il Leitmotiv è più volte menzionato da Gadda

40 Cfr. la nota di Manzotti, p. 110. 
come cardine costruttivo prediletto: cfr. ad es., nella nota del 26 luglio

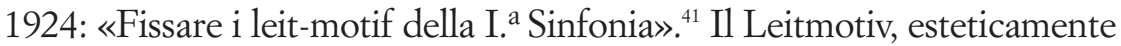
suggestivo, sul piano della struttura produce anche l'effetto di arginare le continue interruzioni, i prorompenti decentramenti, gli inciampi nella frase e nel racconto. Perlopiù insiste su immagini paesistico-naturali elevate a emblemi: come le robinie funestamente invasive, detestate al pari delle pere butirro, o i carpini. $\mathrm{E}$ in generale tocca il vasto bestiario perlopiù presente in chiave comica: le cicale ${ }^{42}$ le mosche, le galline, i polli ( $\ll \mathrm{I}$ polli i polli, gli esemplari polli»), ${ }^{43}$ la tinca, i gatti. Spesso il Leitmotiv coincide efficacemente con profonde ossessioni, che mette in rilievo, collegate a Gonzalo o attribuite al suo punto di vista iroso e angosciato: l'inefficacia del muro di protezione, l'odio contro le campane dove si dispersero le finanze familiari, la sua avarizia-avidità-golosità, il ritratto del padre frantumato (vedi la Mademoiselle de Vinteuil citata da Contini), il «senso feroce ed esclusivo del possesso», l'orologio fracassato, i brillanti della madre, le minacce contro la madre, il conflitto con il peone e con il vigile, il sogno presago della madre morta. Insomma un'officina del «molteplice» e del «complesso», sulla cui genesi e sul cui attento coordinamento, come appare dallo studio di Chiara Ornago, è lecito attendersi ancora dati, e ragioni di riflessione.

\section{LA CASA E LA GUERRA}

La casa della Cognizione è di continuo «maledetta» (p. 301) da Gonzalo in sarcastiche invettive. Come sono messi in satira suoi dettagli o proiezioni sentimentali correlati: la «memoria del padre che l'aveva costruita» (p. 301); il muro «pirobutirrico» (p. 172) inadeguato alla protezione; la smania borghese del possesso, l'aborrito narcisismo, che anche nella severa Adele poterono generare il «trionfo serpentesco della [...]

41 A p. 40.

42 Sulle cicale, cfr. Claudio Vela, Le cicale (e altro bestiario) della Cognizione, online su EJGS (già in volume nel 2005); sulla robinia, cfr. Emilio Manzotti, Una scheda per la robinia, QI, n.s., 2012, 3 (già in EJGS).

43 Appuntato tra le Note costruttive e battute da interpolare, e leggibile nell'ed. commentata della Cognizione, p. 566. 
villa sopra le rivali keltikesi» (p. 302). Nel dialogo Gonzalo-medico, sono attaccati anche $\mathrm{i}$ «luridi» pronomi-«pidocchi» che infestano l'io (pp. 17576); ancora le campane «menadi scaravoltate a pancia all'aria», che assorbirono il patrimonio familiare (p. 178); i fetidi peones zoccolanti per casa; «la conigliera di nipoti» dei vicini (p. 167), accuditi dalla madre con ripetizioni «a gratis» (p. 166); il violinista «nano», ospitato a casa dalla madre e riverito per dabbenaggine (pp. 207-209). ${ }^{44}$

Altro filo conduttore è il trauma della guerra, enunciato nel secondo paragrafo dell'umoristico esordio («Il Maradagàl, come è noto, uscì nel 1924 da un'aspra guerra col Parapagàl», p. 9). La guerra travolse il fratello, ma anche umiliò il protagonista, producendo in GonzaloCarlo sofferenze, rabbie, gravi prove, e risparmiandogli la vita soltanto per amaro e colpevolizzante contrappasso.

Un ultimo punto: nella messa in scena della Cognizione del 2014 all'Out off, regista Loris, straordinario protagonista Mario Sala con Monica Bonomi, colpisce la bellissima idea registica: la scena spoglia e bigia, monocroma, mai modificata, è la casa, ridotta anti-naturalisticamente al minimo, in cui si incorpora la materia della guerra. Della casa appaiono brandelli cadenti e degradati: tracce di cucina, la balaustra del terrazzo, e, appena accennati a livello superiore, gli spazi della solitudine di Gonzalo (studio e letto). I frammenti della casa sono circondati-invasi da cataste di sacchi: la casa è nel contempo la trincea della terribile guerra: casa-trincea, casa-guerra, casa-morte, rovina, distruzione, trauma.

\section{IL «ROMANZO DI UNA VITA»: UN CANTIERE ININTERROTTO}

Ben a ragione Roscioni definisce la Cognizione il «romanzo di una vita»: Gadda ne fu occupato quasi ininterrottamente. Non si consideri

44 Parecchi di questi materiali (la casa di Longone, il ritratto della madre ma in età più giovane, e della domestica che «pisciava [...] all'impiedi») erano già entrati nel racconto del 1929, Villa in Brianza, dove però fu assegnato grande spazio eccezionalmente al padre Francesco (e alla figlia di primo letto, la tagliente Emilia«Madonna Ipoteca»). Villa in Brianza, estratto dal Fondo Roscioni, fu edito a cura di Emilio Manzotti, in QI, 1, 2001, 7-33; e poi da Giorgio Pinotti, in un volumetto adelphiano (Milano, 2007). 
solo il '38, avvio dell'edizione in rivista (e il '37, avvio del lavoro sui primi autografi). Infatti come si sa della Cognizione esistono alcune parziali ma significative prefigurazioni (Villa in Brianza, e I viaggi di Gulliver, cioè del Gaddus), ${ }^{45}$ ne è anticipato il tema-chiave del matricidio (Dejanira), e ne ricorrono già nel Giornale di guerra e di prigionia alcuni temi, come il ritratto della madre figura del sacrificio venerata e insieme oggetto d'ira, e come la presenza della casa di campagna.

$\mathrm{Nel}$ cantiere filologico-critico intrapreso dall'edizione del Racconto italiano di ignoto del novecento (1983) sino a Un fulmine sul 220 (2000), anche con i restauri operati sulla Meccanica e su Dejanira Classis-Novella seconda, ${ }^{46}$ Dante Isella ha fatto emergere testi fondamentali di Gadda e un dato critico inedito che fanno sistema intorno alla Cognizione: la tensione ininterrotta al romanzo, genere della modernità, modello in Italia il Manzoni. Il romanzo appare perseguito senza sosta: dal progetto giovanile esiguo di Retica ${ }^{47}$ attraverso il Racconto italiano del 1924-'25 dove Gadda esplora contenuti e modalità del raccontare; via via, attraverso Dejanira, La meccanica, sino al Fulmine. La cui acquisizione rivela che gli anni trenta sono per intero devoluti alla satira. Basti citare i superbi racconti San Giorgio in casa Brocchi e L'incendio di via Keplero, tra anni trenta e avvio anni quaranta. Ma sono interessantissimi sul versante satirico altri testi minori dell'epoca come il racconto $L a$ casa, «accigliato-umoristico-bisbetico»; ${ }^{48}$ o gli apologhi alla Swift che Isella riunì nell's1 nel volumetto Le bizze del capitano in congedo e altri racconti. ${ }^{49}$ Inerisce pure al registro umoristico l'impegno

45 Viaggi di Gulliver, cioè del Gaddus è datato settembre-ottobre 1933 nella prima e tarda apparizione a stampa, 1970.

46 Raccolte a cura di Isella in Opere, II.

47 Cfr. Paola Italia, Le carte di «Retica», QI, 2, 2003, 295-311, che pubblica i materiali preparatori del romanzo, dedotti dal Fondo Garzanti. Cfr. anche della stessa, Agli albori del romanzo gaddiano: primi appunti su «Retica», in Le lingue di Gadda, Atti del convegno di Basilea, a cura di Maria Antonietta Terzoli, Roma, Salerno, 1995, pp. 179-202.

48 Come si legge in una lettera ad Alberto Carocci, I gennaio 1935, edita in Lettere a Solaria (Roma, Editori riuniti, 1979), e commentata nell'esemplare Nota di Raffaella Rodondi ad Accoppiamenti giudiziosi, in Opere, II, p. 1270.

49 Milano, Scheiwiller, 1981; e Milano, Adelphi, 1981 e $1983^{2}$ (quindi in Opere, II, tra Racconti dispersi e Racconti incompiuti). 
sulle note profuso negli anni trenta e quaranta, nel Castello di Udine del 1934 (per poi farne cadere parecchie, insoddisfatto degli esiti, nell'edizione ultima del '55), e nei racconti dell'Adalgisa.

Sino alla cesura del '36, con la morte della madre. Interviene la concentrazione sulla drammatica Cognizione, che nei recessi di lutto, morte e crimine congiunge traumi personali e collettivi: madre, fratello, guerra, vessazioni emotive, ossessioni, ma è pervasa anche di comicità e grottesco. ${ }^{50}$ Dopo L'Adalgisa ricavata dal Fulmine, subentra il Pasticciaccio, belliano, cruento, polifonico al massimo, farsesco e tragico.

La formula saturnina «romanzo psicopatico e caravaggesco», con cui Gadda definì non tanto il Racconto italiano, quanto il suo ambizioso progetto in una nota stesa in limine del lavoro (14 aprile 1924, p. 31), si potrebbe estendere proficuamente alle sue migliori narrazioni, Cognizione in testa.

50 Per un'analisi della comicità gaddiana, misantropa, accigliata, politicamente scorretta (anche nella Cognizione), cfr. Michele Mari, Lo spasmo comico di Carlo Emilio Gadda, nel Comico nella letteratura italiana, a cura di S. Cirillo, Roma, Donzelli, 2005, pp. 415-431. 
